\title{
Incomplete fractal showers and restoration of dimension
}

\author{
Tatiana Dedovich ${ }^{1, *}$, and Mikhail Tokarev ${ }^{1, * *}$ \\ ${ }^{1}$ Joint Institute for Nuclear Research, 141980 Dubna, Russia
}

\begin{abstract}
The $\mathrm{SePaC}$ and $B C$ methods are used in the fractal analysis of mixed events containing incomplete fractals. The reconstruction of the event distribution by the dimension $D_{F}$ is studied. The procedures of analyzing incomplete fractals and correcting the determination of $D_{F}$ of combined fractals by the $\mathrm{SePaC}$ method are proposed. We find that the $\mathrm{SePaC}$ method fully reconstructs incomplete fractals and suppresses background, the separation of the incomplete fractals and the background by the $B C$ method depends on the basis of the formation of the fractal, and the distribution of events by the value of $D_{F}$ is more accurately reconstructed with the $\mathrm{SePaC}$ method in comparison with the $B C$ method.
\end{abstract}

\section{Introduction}

The interaction of particles at high energies is a complex process. It includes initial and final state interactions of constituents, processes of hard scattering and hadronization. It is often assumed that these processes can be described as showers having a self-similar structure. Let us briefly describe the mechanism of the final-state parton shower used in the PYTHIA Monte Carlo generator [1]. It assumed that the collision of hadrons or nuclei can be described in terms of quarks and gluons. The hard interactions of these constituents are described in the framework of perturbative Quantum Chromodynamics (QCD). The nonperturbative part is model dependent and related with the choice of a parton distribution and fragmentation functions at some factorization, renormalization and fragmentation scales. It is often considered that these scales are equal. After the hard parton-parton scattering, each parton (quark or gluon) can emit one or two partons. The scale of the elementary process is controlled by a transferred momentum. The process looks like a branching parton process in the scale space. In accordance with the momentum conservation law, the secondary partons carry the momentum fractions of initial parton in direction defined by an opening angle for each secondary parton. It is assumed that the secondary parton opening angles are ordered and the branching process has a fractal structure. The final-state showers are time-like. The maximum allowed virtuality scale $Q_{\max }^{2}$ is determined by the hard-scattering process. The virtuality is decreased in each subsequent branching, down to the cut-off scale $Q_{0}^{2}$. It should be noted that the branching threshold for partons leads to the formation of incomplete fractals.

In this work, we consider the reconstruction of incomplete fractals and determination of their dimension. We assume that the hadronization process preserves the fractal structure of a shower. These incomplete fractal showers as well as complete ones are characterized

\footnotetext{
*e-mail: tdedovich@yandex.ru

**e-mail: tokarev@jinr.ru
} 
by the fractal dimension. There are different definitions of a fractal dimension. The fractal dimension $D_{F}$ defined as a value of $d$, which provides a finite value of the Hausdorff measure $M_{H}=\lim _{\delta \rightarrow 0} \sum_{i=1}^{N} l_{i}^{d}$ [2], where $N$ is a number of probes, covering the object with the size $l<\delta$, is called the Hausdorff dimension. Another fractal dimension is the box dimension $D_{b}=\lim _{\delta \rightarrow 0} \ln N / \ln l$ [2]. There are various types of fractals. Among them are fractals with independent [3], dependent [4] and combined [5] partition. The dimensions $D_{F}$ and $D_{b}$ are equal to each other for a fractal with an independent partition and different for dependent and combined fractals.

\section{Fractal analysis}

The fractal analysis is aimed to identify and classify fractals by dimension, separation of the events by fractal types, and reveals new types of events using the classification of fractals. The important task is to chose the phase space in which the object reveals its fractal properties. The other task is the development of an adequate method of analysis. In the present work, we use the Box Counting BC [2] and the System of Equations of P-adic Coverage SePaC [6] methods. The brief description of these methods is given below.

The data sample consists of points with coordinates $X \equiv\left\{x_{i}\right\}$. The procedure of data analysis by the $B C$ method includes the following steps:

1. Reading out the data $X$.

2. Construction of the set of distributions of $X$. (The number of bins $M_{i}$ in distributions changes as a degree of basis two, $M_{i}=(2)^{i}$.)

3. Counting the number of non-zero bins $(N)$ for each distribution.

4. Finding the value of the slope parameter $D_{b}$ of the power function $N \sim M^{D_{F}}$ and the corresponding $\chi^{2}$ of the linear approximation of this function in a double-log scale.

5. Verification of the accuracy condition: if $\chi^{2}<\chi_{\text {lim }}^{2}$ then the set of particles is considered to be a fractal.

The $B C$ method determines the box dimension $D_{b}$ and has only one parameter $\chi_{\text {lim }}^{2}$.

The procedure of data analysis by the $\mathrm{SePaC}$ method includes the following steps:

1. Reading out the data $X$.

2. Construction of the $P$-adic coverages: $P=3, . . P_{\text {Max }}$. Each coverage is a set of distributions of variable $X$. The number of bins $M_{i}$ in the distributions changes as a degree of the basis $P: M_{i}=(P)^{i}$.

3. Counting the number of non-zero bins $N(l e v, P)$.

4. Analysis of a system of equations for verification of the hypothesis of independent/dependent partition includes:

a. Construction of a system of equations for all levels: $\sum_{i=1}^{N_{l e v}}\left(d_{l e v}\right)^{D_{F}^{l e v}}=1$. Here $N_{l e v}$ is a number of levels and $d_{l e v}$ is the length of non-zero ranges for each level.

b. Finding the solution $D_{F}^{\text {lev }}$ of equations for each level using the dichotomy method.

c. Determination of an averaged value of $\left\langle D_{F}^{\text {lev }}\right\rangle$ and deviation $\Delta D_{F}^{\text {lev }}$.

d. Verification of the accuracy condition: if $\Delta D_{F}^{l e v}<D e v$, then the set of particles is considered to be a fractal ( $P$ is a base and $D_{F}=<D_{F}^{l e v}>$ is a fractal dimension).

The $\mathrm{SePaC}$ method allows one to determine the fractal dimension $D_{F}$. It has two parameters, the maximal base $P_{M a x}$ and the deviation Dev. 


\section{Reconstruction of incomplete fractals}

Here we consider the reconstruction of incomplete fractals from mixed events containing $50 \%$ impurities. The incomplete fractal is obtained by following the recursive generating procedure

$$
x_{1}=x_{0}, \quad x_{i}=f_{j}\left(x_{i-1}\right),
$$

where $x_{0}$ is the initial value, $f_{j}$ is the generating function and $j$ is to be equal to 0 or 1 randomly. The functions $f_{1}$ and $f_{2}$ are written as follows:

$$
f_{1}(x)=\frac{1}{P} \cdot x, f_{2}(x)=\frac{P-1}{P}+\frac{1}{P} \cdot x,
$$

where $P$ is the basis of fractal formation. We generated $10^{3}$ independent fractals with $P=$ $3-8$. The number of points $N_{\text {point }}$ in each event is equal to 64 . The $10^{3}$ random sets of points with the same multiplicity were used as a background.

A procedure for analyzing incomplete fractals with the $\mathrm{SePaC}$ method takes into account the verification of the accuracy condition only for three levels.

Fig. 1(a) shows the dependence of the portion Prtn of the events reconstructed as fractals using the $\mathrm{SePaC}$ method on $P_{\text {Max }}$. The portions Prtn for the mixed events including fractals (green, red and blue symbols for $P=9,5,3$, respectively) and background (gray symbols) are significantly different. Starting from $P_{\operatorname{Max}}=5$, the portion for all mixed events is the same. It has a plateau. The choice of $P_{\text {Max }}$ as a minimal value on the plateau allows reconstructing all fractals and suppressing all background $(\operatorname{Prtn}=0)$.

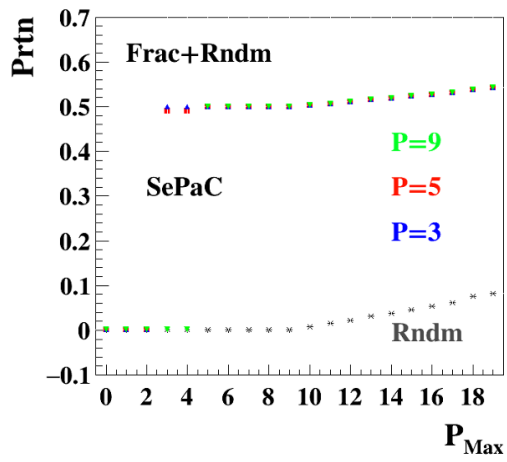

(a)

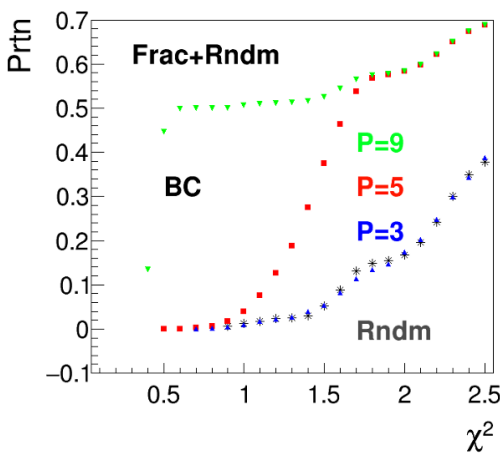

(b)

Figure 1. Portion Prtn of the events reconstructed as fractals using the $\mathrm{SePaC}$ method on $P_{\text {Max }}$ (a) and the $B C$ method on $\chi_{\text {lim }}^{2}(\mathrm{~b})$

Fig. 1(b) shows the dependence of the Prtn of the events reconstructed as fractals using the $B C$ method on $\chi_{\text {lim }}^{2}$. We found that the separation of fractals with $P=3$ (blue symbols) and a background (gray symbols) is not possible because the functions $\operatorname{Prtn}\left(P_{\text {Max }}\right)$ coincide with each other. The choice of $\chi_{\text {lim }}^{2}$ as a minimal value on the plateau allows one to separate fractals with $P=5,9$ (red and green symbols) and a background. The data set extracted from the mixed events and containing fractals with $P=5$ includes a background (about 15\%).

\section{Reconstruction of event distribution by dimension}

Further we describe the reconstruction procedure of the event distribution by fractal dimension. We used the data set of 2001 fractals included an equal number of incomplete in- 
dependent fractals, complete dependent and combined fractals. The two-step procedure of the $\mathrm{SePaC}$ method developed in [7] is used in the analysis. The first step of the procedure includes an additional condition for number of non-zero bins on the first and other levels $N_{i}=\left(N_{1}\right)^{i}$. At the second step, the analysis is carried out without this condition. We found that the dimension $D_{F}$ for independent and dependent fractals is exactly reconstructed and for fractals with combined partition is not reconstructed at the first step.

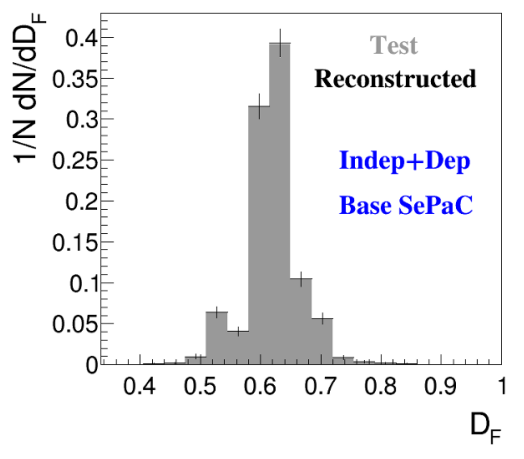

(a)

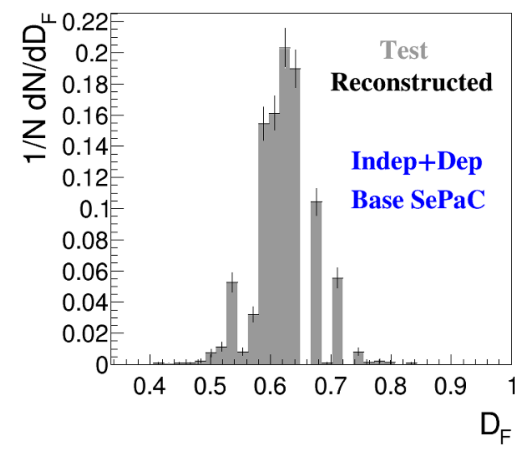

(b)

Figure 2. The event distribution by dimension $D_{F}$ for independent and dependent fractals (gray area) and reconstructed distribution using the base $\mathrm{SePaC}$ method (black cross) at various bin width

Fig. 2 shows the event distributions by $D_{F}$ for independent and dependent fractals at various bin width. One can see that test and reconstructed distributions coincide with each other. At the second step, the fractals with combined partition are analyzed using the $\mathrm{SePaC}$ method without an additional condition.

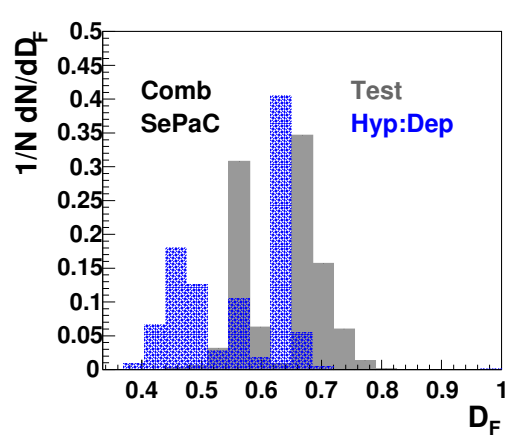

(a)

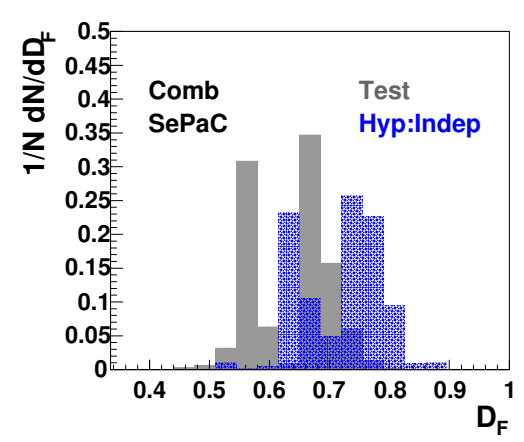

(b)

Figure 3. The event distributions by dimension $D_{F}$ for combined (gray area) and reconstructed (blue area) fractals. The hypotheses of dependent (a) and independent (b) partition of the $\mathrm{SePaC}$ method are used

Fig. 3 shows the event distributions by $D_{F}$ for combined fractals. The hypotheses with dependent (a) and independent (b) partition were used for reconstruction. One can see that for the hypothesis of dependent partition (Fig. 3(a)), the reconstructed distribution is shifted toward smaller values of $\left(D_{F}^{D e p}<D_{F}^{\text {test }}\right)$ and does not retain its shape. The event distribu- 
tion reconstructed using the hypothesis of independent partition (Fig. 3(b)) is shifted toward higher values of $\left(D_{F}^{\text {Indep }}>D_{F}^{\text {test }}\right)$ and retains its shape.

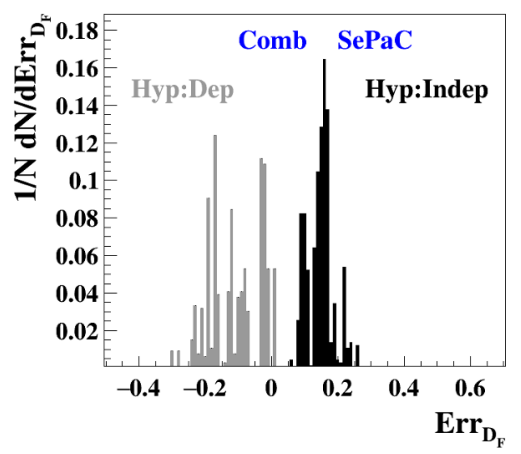

(a)

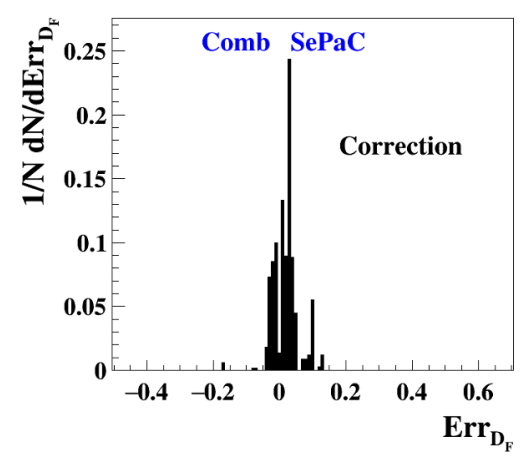

(b)

Figure 4. The event distribution by errors $E r r_{D_{F}}$ obtained using the dependent (gray area), independent (black area) hypotheses of the $\mathrm{SePaC}$ method before (a) and after corrections (b)

Fig. 4(a) shows the distribution of the errors $\operatorname{Err}_{D_{F}}$ obtained using the hypotheses of dependent and independent partition. We see the different displacement of distributions for dependent $\operatorname{Err}_{D_{F}}<0$ and independent $\operatorname{Err}_{D_{F}}>0$ partitions. The errors were found to reach about $30 \%$ and $20 \%$ in these cases. To reduce $E r r$, the correction of the dimension $D_{F}$ was applied. We redefine the dimension obtained by independent partition by the following formula:

$$
D_{F}^{\text {Indep }}=D_{F}^{\text {Indep }}-\left(<D_{F}^{\text {Indep }}>-<D_{F}^{D e p}>\right) / 2,
$$

where $<D_{F}^{\text {Indep }}>$ and $<D_{F}^{\text {Dep }}>$ are averaged values of the dimension obtained by independent and dependent partition, respectively. Fig. 4(b) shows the distributions of the errors $\operatorname{Err}_{D_{F}}$ obtained after the correction. We found that errors decreased, are symmetric around zero and do not exceed $12 \%$.

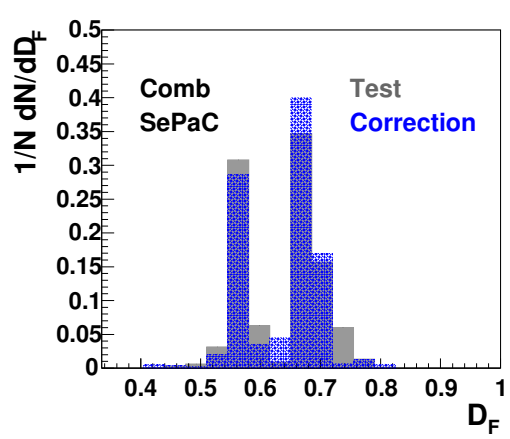

(a)

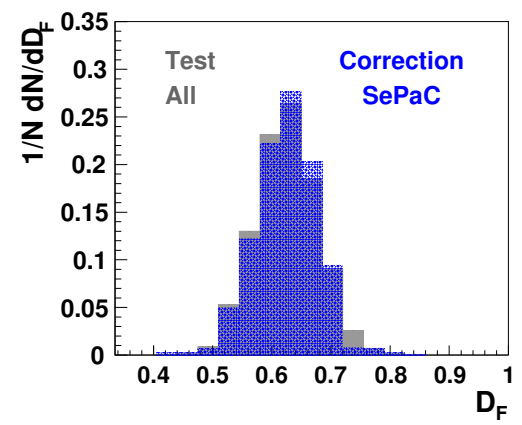

(b)

Figure 5. The event distributions by dimension $D_{F}$ for combined fractals (a) and the full data set (b). The gray and blue areas correspond to the test and reconstructed distributions using the $\mathrm{SePaC}$ method after the correction, respectively

Figs. 5(a,b) show the event distributions by $D_{F}$ for combined fractals and the full data set including incomplete independent fractals, complete dependent and combined fractals. 
The gray and blue areas correspond to the test and reconstructed fractals, respectively. The reconstructed distribution by $D_{F}$ is close to the test one in both cases.

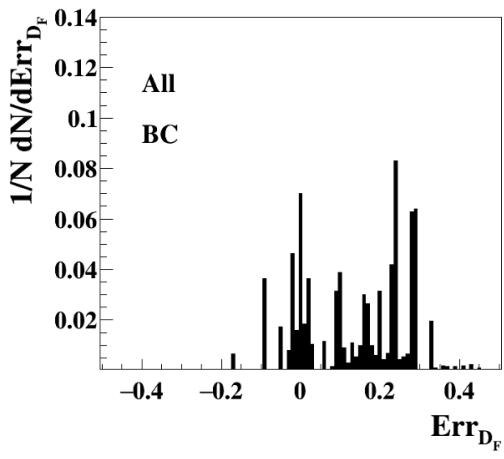

(a)

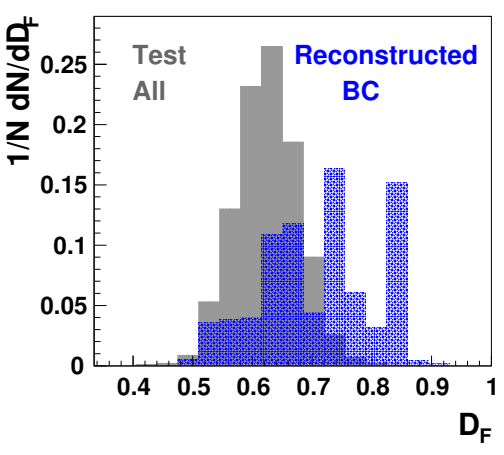

(b)

Figure 6. The event distributions by errors $\operatorname{Err}_{D_{F}}$ (a) and by dimension $D_{F}$ (b) obtained using the $B C$-method for the full data set (the gray and blue areas correspond to the test and reconstruction distributions, respectively)

As seen from the event distribution by errors $\operatorname{Err}_{D_{F}}$ obtained using the $B C$ method (Fig. 6(a)), the errors for $D_{F}$ can reach $40 \%$. Fig. 6(b) shows the event distribution by dimension $D_{F}$ for the full data set (gray area) and reconstructed using the $B C$ method (blue area). We found that the reconstructed distribution is shifted toward the range of larger errors and does not retain its shape.

\section{Conclusions}

The $S e P a C$ and $B C$ methods were used in the fractal analysis of mixed events containing incomplete fractals and reconstruction of the event distribution by $D_{F}$. The procedures to analyze incomplete fractals and correct the determination of $D_{F}$ of combined fractals in the framework of the $\mathrm{SePaC}$ method were proposed. We found that the $\mathrm{SePaC}$ method reconstructs all incomplete fractals and suppresses the background, the separation of incomplete fractals and a background using the $B C$ method depends on the basis $P$ and the event distribution by $D_{F}$ is more precisely reconstructed with the $S e P a C$ method in comparison with the $B C$ method.

\section{References}

[1] T. Sjöstrand, S. Mrenna and P. Skands, JHEP 0605, 026 (2006). hep-ph/0603175

[2] B. Mandelbrot, The Fractal Geometry of Nature (Freeman, San Francisco, 1982) 497

[3] T.G. Dedovich, M.V. Tokarev, Phys. Part. Nucl. Lett. 10, 481 (2013)

[4] T.G. Dedovich, M.V. Tokarev, Phys. Part. Nucl. Lett. 10, 491 (2013)

[5] T.G. Dedovich, M.V. Tokarev, Phys. Part. Nucl. Lett. 2, 169 (2016)

[6] T.G. Dedovich, M.V. Tokarev, Phys. Part. Nucl. Lett. 9, 552 (2011)

[7] T.G. Dedovich, M.V. Tokarev, Phys. Part. Nucl. Lett. 2, 178 (2016) 